\title{
Toxic Effect of Babesiosis in Cattle and Chemotherapiotic Treatment in Egypt
}

\author{
Ahlam F. Hamoda ${ }^{1}$, Mervat Radwan ${ }^{2, *}$, Rabab Rashed ${ }^{3}$, Aziza Amin ${ }^{4}$ \\ ${ }^{1}$ Department of Forensic Medicine and Toxocology \\ ${ }^{2}$ Department of Vetrinary Medicine \\ ${ }^{3}$ Department of Pharmacology, Hospital, Banha University \\ ${ }^{4}$ Department of Pathology Banha University \\ *Corresponding author: maya_salah11@yahoo.com
}

Received August 12, 2014; Revised September 18, 2014; Accepted October 24, 2014

\begin{abstract}
Babesia bigemina plays an important role in causing liver and kidney dysfunction in affected animals. The aim of this study evaluating the toxic effect of babesiosis on liver and kidney by measuring biochemical parameters and pathological tissue changes in infected animals with B. Bigemina and chose the best method of treatment. A total of 40 cattle age 1-3 years 30 were suffered from increase in temperatures, off food, Hemoglobin urea, red water from endemic area with babesia. Take sample from infected animals \& examine it with microscopic examination found babesia bigemina and confirm by inoculation of heparinized blood from this animals in rats and found rats death at fifth day from infection and histopathological exam found babesia in piroplasma form in pathological tissue of rat's liver \& kidney. The infected animals were divided into three groups each group 10 animals untreated group use as control positive and treated groups divided into two groups first group treated with imidocarb only and the second group use imidocarb and lincomcyn as antibiotic drug and compare between two groups by measuring liver\& kidney function improvement and compare two groups with negative and positive group which method of treatment more effective in treatment of babesia \& decrease toxic effect of babesia.
\end{abstract}

Keywords: cattle, babeseosis, histopathological changes in expermintal infected rat

Cite This Article: Ahlam F. Hamoda, Mervat Radwan, Rabab Rashed, and Aziza Amin, "Toxic Effect of Babesiosis in Cattle and Chemotherapiotic Treatment in Egypt.” American Journal of Infectious Diseases and Microbiology, vol. 2, no. 5 (2014): 91-96. doi: 10.12691/ajidm-2-5-1.

\section{Introduction}

This review deals primarily with the treatment of babesiosis in cattle. It is thought that these animals are the most severely affected by Babesia infection from an economy point of view, [1]. Present anemia may attributed to, Immunomediated phenomena by which autoantibodies directed against component of membrane of infected and uninfected erythrocytes, production of toxic hemolytic factors of the parasite, mechanical damage by trophozoite intra erythrocytes binary fission. erythrophagocytosis and through of release vasoactive molecules such as kallikrein $[2,3,4]$, and [5]. On the other hand, according to the role of liver in lipidbiosynthesis, it can be argued that liver may play an important compensatory role in balancing lipid components in blood flow to prevent anemia during bovine tropical theileriosis [6]. Babesiosis is disease worled-wide distribution affecting many species of mammles principally cattle and man. major impact occurs in the cattle industry where bovine babesiosis has had ahuage economic effect due to loss of meat and beef production of infected animals and death [7] and [8]. Now days to those costs must be added the costs of tick control. There are limited chemotherapeutic choices reliable and fast detection methods. Detection and treatment are important tools to control babesiosis. Microscopic examination still cheapest and fastest methods used to identify babesia parasites [9] and [10].

\section{Material and Methods}

A total of 40 cattle age 1-3 years. 30 were suffered from increase in temperature, off food, hemoglobin urea, red water from endemic area with babesia. Take two samples from infected animals first with anticoagulant for blood smear and experimental infection by inoculation in rats and second sample to separate serum to determined biochemical changes associated with babesia Bigemina infection in cattle as (Fe, NO, SOD, Cholesterol, Triglyceride, BUN, Creatinine, GOT, GPT and TAC) according to [11]. By microscopic examination found positive to babesia Bigemina, babesia Bigemina is large in size and can extend in the full diameter of an erythrocytes, diagnosis of babesiosis are made by examination of three blood film with Giemsa for the best results. Blood films should be prepared from capillary blood collected for instance, after pricking the margin of an ear and confirm microscopic examination with inoculation of heparinized blood in experimental animals as rats transemation test 
was performed in Rats groups by challenging them with 1 $\mathrm{ml}$ of infected haparenized blood (5x106 babesia Bigemina) infected parasitic erythrocytes were maximum at 5 days post experimental infection so all rats died, when compare with control group clinical signs appear at fourth day of infection, histopathological sections of inflammation of rats were done and indicated that the liver and kidney were severely affected, according to this test the infected cattle were divided into three groups each group consists of 10 animals, treated groups divided into two groups first group treated with imizol only at dose (Imizol $12 \%-1 \mathrm{ml} / 100 \mathrm{~kg}$ by s/c injection) and the second group use imizol and lincomycin as antibiotic at dose (1.2 g paranterally, twice daily for seven or ten days and third untreated group use as control positive.

\section{Results}

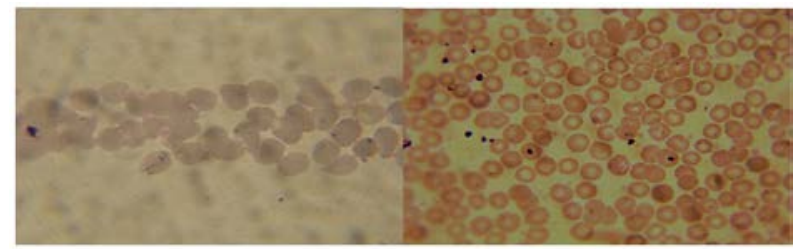

Figure 1. Blood smear show different shapes of babesia bigemina shizonts, aclassic tetrad in anRBCs of expermintal infected rat arranged in the form of maltase cross and pyriform shapes (wright-Giemsa,x100)
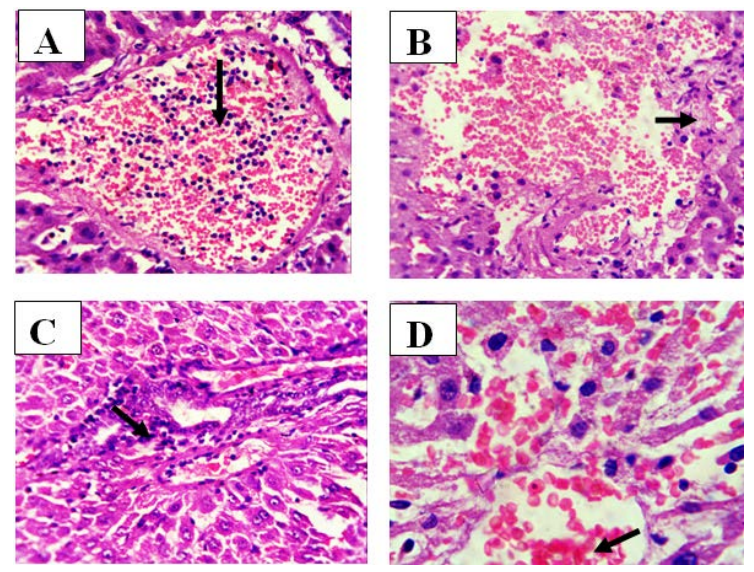

Figure 2.

Liver of rats experimentally infected with babesia showing (A), severe congestion of hepatic blood vessels with the presence of eosinophils, lymphocytes and macrophages (arrow, x200), (B) hemorrhagic area scattered in the hepatic parenchyma accompanied by destruction of some hepatic cells (arrow, x200), (C) periportal leukocytic cellular infiltrations with mild hyperplasia of the bile duct (arrow, x200), (D) babesia parasitized RBCs inside the hepatic blood vessels (arrow, x1000). H\&E stain
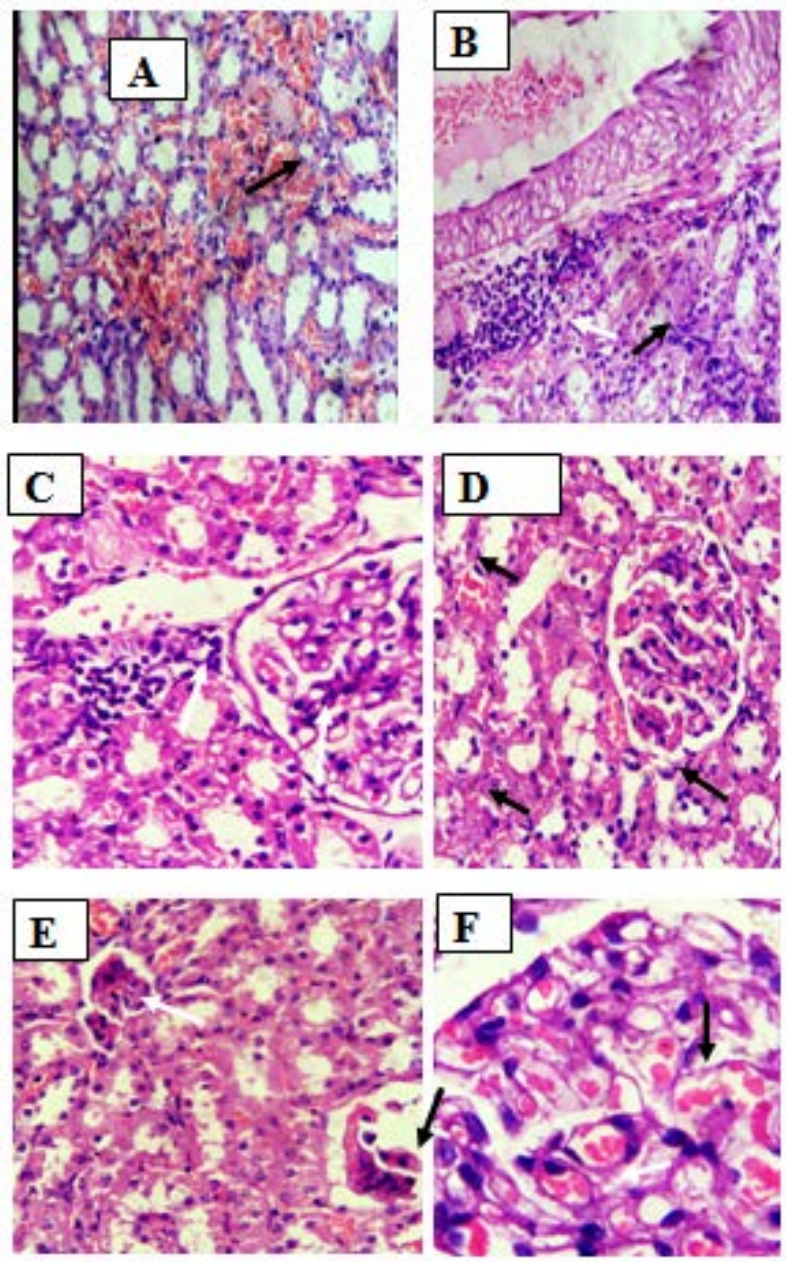

Figure 3.

Kidney of rats experimentally infected with babesia showing (A), severe congestion of inter-tubular blood capillaries in the renal medulla (arrow, x200), (B) perivascular mononuclear leukocytic cellular infiltrations (white arrow) with degenerative changes in the cytoplasm of the lining epithelium of the renal tubules (black arrow, x200), (C) hypersegmentation of the glomerular tuft (white arrow). Note also vacuolation of the lining epithelium of the renal tubules (black arrow, x200), (D) vesiculation of the endothelial lining of the capillary tuft of the glomeruli with periglomerular edema (black arrow) and leukocytic cellular infiltration (white arrow, x200), (E) shrinkage of the glomerular tuft (white arrow) or even complete destruction of the glomerular tuft (black arrow, x200), (F) babesia parasitized RBCs inside the glomerular capillaries (arrow, x1000). H\&E stain.

Table 1

\begin{tabular}{|c|c|c|c|c|}
\hline Groups Parameter & Control Negative & Control Positive & Treat 1 Imiizol & Treat 2 Imizol and clidamycin \\
\hline Fe & $1.650 \pm 0.2458^{\mathrm{a}}$ & $0.5733 \pm .6488^{\mathrm{b}}$ & $0.660 \pm 0.064 .8^{\mathrm{ac}}$ & $1.222 \pm 0.1080^{\mathrm{a}}$ \\
\hline NO & $11.08 \pm 0.551 \mathrm{a}$ & $44.44 \pm 3.55^{\mathrm{b}}$ & $29.84 \pm 3.286^{\mathrm{ac}}$ & $25.69 \pm-2.55^{\mathrm{a}}$ \\
\hline SOD & $25 \pm 0.57^{\mathrm{a}}$ & $9.317 \pm 0.4126^{\mathrm{b}}$ & $10.70 \pm 0.2467^{\mathrm{ac}}$ & $14.53 \pm 1.677^{\mathrm{a}}$ \\
\hline Cholesterol & $110 . \pm 10.11^{\mathrm{a}}$ & $183.3 \pm 5.696^{\mathrm{b}}$ & $126.2 \pm 10.01^{\mathrm{ac}}$ & $123.3 \pm 3.48^{\mathrm{a}}$ \\
\hline Triglyceride & $57.67 \pm 1.202^{\mathrm{c}}$ & $94.00 \pm 7.00^{\mathrm{ac}}$ & $46.2 \pm 12.08^{\mathrm{a}}$ & $39.17 \pm 3.027^{\mathrm{b}}$ \\
\hline Urea (BUN) & $30.40 \pm 3.881^{\mathrm{a}}$ & $51.80 \pm .028^{\mathrm{ac}}$ & $42.60 \pm 5.437^{\mathrm{a}}$ & $32.80 \pm 2.782^{\mathrm{b}}$ \\
\hline Createnine & $0.8800 \pm 0.07767^{\mathrm{a}}$ & $2.197 \pm 0.2867^{\mathrm{ac}}$ & $1.111 \pm .06445^{\mathrm{a}}$ & $1.157 \pm 0.02899^{\mathrm{b}}$ \\
\hline GOT & $63.40 \pm 6.562^{\mathrm{c}}$ & $154.7 \pm 8.090^{\mathrm{bc}}$ & $101.2 \pm 7.049^{\mathrm{b}}$ & $82.67 \pm 9.7801^{\mathrm{ac}}$ \\
\hline GPT & $25.8 \pm 2.131^{\mathrm{a}}$ & $59.67 \pm 4.417^{\mathrm{b}}$ & $54.67 \pm 3.160^{\mathrm{bc}}$ & $37.00 \pm 6.11^{\mathrm{a}}$ \\
\hline TAC & $2.823 \pm 0.6041^{\mathrm{a}}$ & $1.410 \pm 0.2631^{\mathrm{c}}$ & $1.987 \pm 1.062^{\mathrm{b}}$ & $2.143 \pm 6.3123^{\mathrm{a}}$ \\
\hline
\end{tabular}


Figure 1 showed that different forms of shizonts of babesia bigemina in RBCs of infected rat which challenge with infected blood from cattle naturally infected with babesia Bigemina this test confirm diagnosis babesia Bigemina in these animals to help treatment and control, The microscopically examination of various organs of rats experimentally infected with babesia bigemina revealed various histopathological changes in different organs such as liver, kidneys. The examined livers showed severe congestion of hepatic blood vessels and blood sinusoids. The blood vessels were enriched with high number of eosinophils, lymphocytes and macrophages (Figure 1A). Focal areas of hemorrhages in between degenerated hepatocytes and accompanied by destruction of few hepatic cells were also observed (Figure 1B). The Vonkupffer cells are enlarged. Furthermore, peri-portal as well as peri-vascular mononuclear leukocytic cellular infiltrations mainly lymphocytes and macrophages was demonstrated, in association with mild hyperplasia of the bile duct (Figure 1C). Additionally, babesia was found parasitized the erythrocytes (Figure 1D). Histological abnormalities were detected in the kidneys of rats experimentally infected with babesia. Severe congestion of the inter-tubular blood capillaries in the renal medulla was demonstrated (Figure 2A). Furthermore, most of the investigated kidneys were destructed, as the renal tubules showed degenerative changes in the cytoplasm of the lining epithelial cells in association with perivascular mononuclear leukocytic cellular infiltrations mainly lymphocytes and macrophages (Figure 2B). Additionally, hyaline casts were observed in the lumen of renal tubules together with accumulation of edematous fluid was also noted in the renal tissue with destruction of the basement membrane of some renal tubules. Furthermore, vacuolation of the lining endothelial cells of the glomerular capillaries and Peri-glomerular edema and leukocytic cellular infiltrations were demonstrated (Figure 2C). Occasionally, hypersegmentation of the glomerular tuft was also noticed (Figure 2D). However, edema and thickening in the wall of the capillary of glomerular tuft were also detected. Occasionally, fusion of the glomerular tuft with the parietal layer of Bowman's capsule was seen. Sometimes, various pathological changes were observed in the glomeruli such as shrinkage of the glomerular tuft or even complete destruction of the glomerular tuft (Figure 2E). Interestingly, babesia was found parasitized RBCs inside the renal blood vessels as well as inside the glomerular capillaries (Figure 2F).

Table 1 showed that significant different Values are expressed as mean and mean of standard error, values with different superscriptions ( $a, b$ and $a b$ ) in rows differ significantly $(\mathrm{P}<0.05)$. In general infected animals had lower values of antioxidant parameters as(TAC, SOD and $\mathrm{Fe}$ ) when compared with healthy animals. On other side value of oxidant markers (NO) significant increase in blood of infected group and significant different in Cholesterol, Triglyceride, Urea (BUN), GOT and TAC when compare healthy group. When compare between control groups (negative and infected) and treated groups (treated with Imizol only GI and Imizol associated with clindamycine antibiotic GII) found significant changes among four groups in antioxidant and oxidant parameters. When compare groups with healthy group found significant different increase oxidants and significant different decrease antioxidants enzymes activity among infected group, treated group with Imizol only and non significant different between healthy group and treated group with imizol and

\subsection{Statistical Analysis}

The results were analyzed by a one-way analysis of variance (ANOVA) followed by the Duncan test for multiple comparisons using computer software, Duncan's Multiple Range Test.

\section{Discussion}

Tick fever or cattle fever (babesiosis) is economically the most important arthropod-borne disease of cattle worldwide Our study revealed that kaliobia governorate endemic with Babesia Bigemina and when examine animals aged from 1-3 years suffered from increase temperature emaciated, off food, bloody urine and by blood smear found babesia Bigemina after an infectious tick bite [12] The parasites invade red blood cells and first type of trophozoite differentiates, replicating asexually by budding with the formation of two to four merozoites tetrad formation. A second type of undifferentiated trophozoite is also formed that does not replicate but enlarges and differentiates into gametocyte-like forms similar to that seen in Plasmodium species. Merozoites eventually disrupt infected erythrocytes and reinvade other red blood cells the presence of tetrad formation often is mentioned as a diagnostic aid, trophozoites may be seen outside erythrocytes extracellular forms, which suggests the possibility of transmission by plasma and platelet products with Babesia infection this came agree with $[13,14,15,16]$ and [17] who concluded that three distinguishing features differentiate the Babesial organisms usually form tetrads ("Maltese cross"), do not have hemozoin pigments within the affected red blood cells and have extracellular merozoites,. Our investigation confirm infection with babesia by intraperitoneal inoculation of $1.0 \mathrm{~mL}$ of heparinized-anticoagulated whole blood of diseased animals into the peritoneum of rats followed by smear positively and rats death at fifth days from infection after clinical sings appear, this results came agree with [18] and [19] who reveald that when confirm babesia infection by inculcation of heparinzed blood from infected animals in rats found that rats death in 5 days post infection with clinical signs appear on it from fever parasetemia shivering, bloody urine progressive rise in rectal temperature to 40c \& death to animals. when compare with control, in histopathological showed that different forms of shizonts of babesia Bigemina in RBCs of infected rat which challenge with infected blood from cattle naturally infected with babesia Bigemina this test confirm diagnosis babesia Bigemina in these animals to help treatment and control, The microscopical examination of various organs of rats experimentally infected with babesia bigemina revealed various histopathological changes in different organs such as liver, kidneys, testes, intestine and brain. The examined livers showed severe congestion of hepatic blood vessels and blood sinusoids. The blood vessels were enriched with high number of eosinophils, lymphocytes and macrophages, Focal areas of hemorrhages in between 
degenerated hepatocytes and accompanied by destruction of few hepatic cells were also observed, The Von-kupffer cells are enlarged. Furthermore, peri-portal as well as perivascular mononuclear leuocytic cellular infiltrations mainly lymphocytes and macrophages was demonstrated, in association with mild hyperplasia of the bile duct, Additionally, babesia was found parasitized the erythrocytes, Histological abnormalities were detected in the kidneys of rats experimentally infected with babesia. Severe congestion of the inter-tubular blood capillaries in the renal medulla was demonstrated, Furthermore, most of the investigated kidneys were destructed, as the renal tubules showed degenerative changes in the cytoplasm of the lining epithelial cells in association with perivascular mononuclear leukocytic cellular infiltrations mainly lymphocytes and macrophages, Additionally, hyaline casts were observed in the lumen of renal tubules together with accumulation of edematous fluid was also noted in the renal tissue with destruction of the basement membrane of some renal tubules. Furthermore, vacuolation of the lining endothelial cells of the glomerular capillaries and Periglomerular edema and leukocytic cellular infiltrations were demonstrated, Occasionally hyper segmentation of the glomerular tuft was also noticed, However edema and thickening in the wall of the capillary of glomerular tuft were also detected Occasionally fusion of the glomerular tuft with the parietal layer of Bowman's capsule was seen. Sometimes, various pathological changes were observed in the glomeruli such as shrinkage of the glomerular tuft or even complete destruction of the glomerular tuft. Interestingly, babesia was found parasitized RBCs inside the renal blood vessels as well as inside the glomerular capillaries. This results agree with [20] who found that rats death at fifth day from infection and histopathological exam found babesia in piroplasma form of babesia in pathological tissue of rat's liver \& kidney, Hyperplasia of Kupffer cells were detected in liver sections of infected gerbils, this is due to the need of phagocytosis as a protective mechanism during the course of infection and babesia parasite, like theleria and malaria parasites, invade erythrocytes of infected animals resulting in destruction of parastized erythrocytes. Immune response of gerbils due to Babesia rodhaini infection,. hepatic tissue showed cytoplasmic vacuolation which is mainly a consequence of considerable disturbances in lipid inclusions and fat metabolism occurring under pathological cases [21]. Our study investigated significant changed in lipid profil synthesize in liver as cholesterol and triglyceride due to toxic effect of babesia on liver when compare control positive with control negative and treated groups. these results were agree with [22] who reported a marked increase in cholestrolemia in experimentally $\mathrm{T}$. annulatainfected cattle and stated that this increase was probably due to liver damage and this investigation agree with [23] who concluded that insulin concentration in dog with babesiosos is low and metabolism of adipose tissue is strictly related to insulin, Consequently, reduce level of insulin during babesiosis seems other reason for elevation of cholesterol and triglycerides content and agree with(Angle Tomas et. al. 2005 [24] who reported that cholesterol and triglecerides concentration higher for infected dog with renal azotemia result from babesia infection which lead to liver compensatory reaction to the loss of proteins, an increase production of proteins including the lipid transport proteins, but differ with (Okubo 2007 [25] who concluded that cholesterol is a major component of the erythrocyte membrane erythrocyte invasion and replication by $\mathrm{B}$. bigemina are affected by the amount of cholesterol in the host erythrocyte membrane (Bijan Esmaeilnejad 2012 [26] who recorded that and reported that, cattle clinically infected with blood parasite had significantly lower serum total protein, calcium, cholesterol and triglecerides concentration and the lipids are the major part of red blood cells membrane, the unchanged serum lipid composition and cholesterol in the infected animals could imply that the plasma lipid concentration probably have not a marked responsibility for the increased fluidity and permeability of Theileria annulata in infected erythrocytes. Our study investigated increase in GPT and GOT enzymes when compare control positive with control negative and treated groups and when evaluate TAC, SOD, Gsh, and Fe as antioxidants found significant decrease when compare control positive with control negative and treated groups and significantly higher alkaline phosphate (ALP) alanine aminotransferase (ALT) and aspartate aminotransferase (AST) activity, phosphorus, sodium, potassium, bilirubin (direct and indirect) and (Otsuk, et. al 2002 [21] who concluded that the activietes of SOD and TAC and the concentration of GSH were low Hypoglycaemia such as increased glucose consumption, depletion of hepatic glycogen stores, and hepatic dysfunction with impaired gluconeogenesis [27]. Acute hepatopathy was detected in all dogs with elevated ALT activity in animals infected with babesia while [23] observied that Babesia species produce haemolysis of erythrocytes so lead to production of erythrocytes from bone marrow to compensate decrease in erythrocytes level, this lead to decrease serum level of iron which is necessary for erythrocyte production. Our study found significant changes in kidney functions tests as(BUN \& createine) which increase in infected animals when compare with healthy and treated animals this agree with [21] significantly higher blood urea Nitrogen (BUN) concentrations than the healthy cattle. [28,29] and [30] Renal function also is affected occasionally and Interleukin-1 release. [23] Observed elevation in BUN and creatinine level might have resulted from kidney dysfunction, muscle catabolism, and colonization of B. ovis in the renal blood circulation. It is suggested that in ovine babesiosis many potential factors leading to impairment renal function. In our study most of the investigated kidneys were destructed, as the renal tubules showed degenerative changes in the cytoplasm of the lining epithelial cells in association with perivascular mononuclear leukocytic cellular infiltrations mainly lymphocytes and macrophages, acute diffuse proliferative glomerulitis, acute glomerular hemorrhage, presence of thrombi, congestion and stasis in glomerular capillaries, acute glomerular hemorrhage and acute tubular necrosis. Main observed histopathological changes in kidneys in naturally acquired B. canis infected were vacuolarhydropic degeneration, necrosis, detachment of renal tubular epithelial cells in proximal convoluted and hemoglobin casts. Moreover; hypoxia appears to be more important than hemoglobinuria in damaging kidney of experimentally and naturally Babesia infected dog Mathe et. al. 2007 [31] it seems that elevation in BUN and creatinine level ascribes to kidney malfunctions in 
infected small ruminants with babesia.. when compare control groups with treated groups found group treated with antiparasitic imizol and clindamycin is the best group than other groups in results \& propagation since return to normal faster. When compare among treated groups which return to normal faster and all functions test return to normal when compare with control groups. This study agrees with [32] and [33], Imizol has been successfully used as a chemo prophylactic, Exchange transfusion, together with antibabesial chemotherapy, may be necessary in critically ill patients. This treatment is usually reserved for patients who are extremely ill with blood parasitemia of more than 10 percent, massive haemolysis and asplenia and [34] and [35] babesiosis may continue for more than two months after treatment and when left untreated, silent babesial infection may persist for months or even years when compared the duration of parasitemia in persons who had not been treated. Babesial DNA persisted for 16 days in 22 actually ill subjects who received clindamycin and quinine therapy but among the subjects who did not receive specific therapy symptoms of babesiosis persisted up to 114 days, clindamycin is promising and may become prominent in the future Antibabesial chemotherary \& Antibiotic necessary in critically, ill patients. This treatment is usually reserved for patients who are extremely ill- with blood parasitemia. Clindamycin, the treatment of choice for Babesia microti, the chief Babesia species that infects humans, can be used against Babesia in dogs. since this is a readily available antibiotic and the drugs specific for Babesia, clindamycin represents an excellent starting point for therapy In clinical cases, a combination of clindamycin and quinine is administrated as the standard treatment [18] and [36]. Lincomycin and clindamycine are the only members of the relatively small lincosamide antimicrobial class marketed for use in humans clindaycin gradually reduced parasitemia levels and induced morphological changes in parasites that indicate degeneration of parasites as segmentation size reduction, localization in the cell limbic, tornstate of nucleus and disappear of cytoplasm, clindamycin treatment reduce clinical signs of babesia infection, it still enjoys use in the therapy and prophylaxis of a large number, protozoa [1] and [37].

\section{Conclusion}

To our knowledge, The observed increase BUN and creatinine indicate renal failure and increase in GPT, GOT and decrease lipids profile (triglycerids and cholesterol) indicate disturbance in liver function and oxidative strees associated with infection as SOD TAC and Fe came in agreement with those reported earlier. Anorexia in relation to high rise of body temperature.

\section{Acknowledgment}

Acknowledgment to all member stuff of education veterinary hospital Benha University wherever the experiment were performed and stuff of department of infectious diseases special dr Mohamed hassanin Ebeid prof. of infectious diseases Department of Veterinary Medicine.

\section{References}

[1] Henri J. Vial *, A. Gorenflot Chemotherapy against babesiosis Veterinary Parasitology 138 (2006) 147-160.

[2] Trotta M, Carli E,Novari G, clinicopathological findings, moleculer detection and characterization of babesia gibsoni infection in a sack dog from italy vet-parasitol 2009, 165-318-322.

[3] Rubino G,Cito Am, Lacinior, Hematology and some blood chemical parameters as function of tick-borne disease signs in hors. J Equine Vet. Sci 2006: 26: 475-480.

[4] Rafag RB, Mrljk V, KucerN et.al. Protein C activity in babesiosis of dogs, Vet. Arhir, 2007, 77, 1-8.

[5] ZobbaR Adru M, Niccolini S, et. al. Clinical laboratory finding in equine piroplasmosis, J.equine Vet. Sci. 2008, 28, 301-308.

[6] Kamlie BicRk, yeten Deger, Sedar Deger, some biochemical and haematological parameters of sheep infected with babesia species YYU vet Fak Derg 2005,16 (1): 33-35.

[7] Omer O.H., EL-MALIK K.H, MAGZOUB M. MAHMOUD O.M., HAROUN E.M., HAWAS A., OMAR H.M.: Biochemical profiles in Friesian cattle naturally infected with Theileria annulata in Saudi Arabia vet. Res. Comm., 2003, 271-25.

[8] YADa C.K. Shurma N.N, changes in blood chemical compoents during experimentally in duced Theileria annulate infections in cattle Vet. Parasitol 1986; 21, 91-98.

[9] Mosqueda J,Olevera-Ranirez A, Aguilar-Tipacamu GJ Current advances in detection and treatment of babesiosis. Curr Med Chem.2012: 19 (10), 1504-18.

[10] Bock R. L.Dackson, A.DE vos and W.Jorgensen. Babesiosis of cattle parasitology (2004), 5247-5269.

[11] Koracevic, D, Koracevic,. G. et al, Total Antioxidant Capacity. J Clin Pathol. 54, 356-361 (2001) El-Aaser, A.A. (unpublished).

[12] Spach DH,Liles WC, Campbell GL, quick RE, Anderson DE, fritsche TR. Tick-borne diseases in the united states. N Engl J Med. 1993; 30: 936-47.

[13] El Sawalhy, A. A. (1999): "Veterinary Infectious Diseases" $2^{\text {nd }}$ Edit. Ahram Distribution Agency, Egypt.

[14] Sumamsetty, MD, PHD, Zena Khalil MD,Pamela, Schori,MT (ASCP), Migul Azar, MD, Babesiosis two atypical cases from minnestota and review, Am. J. Clin. Pathol. 2003: 120,554-559.

[15] Gelfand JA. Babasia. In: Mandell GL, Douglas RG, Bennett JE, DolinR, eds. Mandell, Douglas and Bennett's, principles and practice of infectious diseases. $5^{\text {th }}$ ed. New York: Churchill Livingstone, 2000; 2899-902.

[16] Pruthi RK, Marshall WF, Wiltsie JC, Persing DH. Human babesiosis. Mayo Clin Proc. 1995; 70: 853-62.

[17] Boustani MR, Gelfand JA. Babesiosis. Clin infect Dis. 1996; 22: 611-5. Cats. Afr.vet.ver. (2000) 71 (1): 53-5.

[18] Bonoan JT, Johnson DH, Cunha BA. Life-threatening babesiosis in an asplenic patient treated with exchange transfusion, azithromycin, and atovaquone. Heart Lung. 1998; 27: 424-8.

[19] Loutan L, Rossier J, Zufferey G, Cuenod D, Hatz C, Marti HP, Gern L. Imported babesiosis diagnosed as malaria [Letter]. Lancet. 1993; 342: 749.

[20] DKhil,M.A. S.AL. Quraishy, A.S. Abdel Baki. Hepatic tissue damage induced in meriones ungliculatus due to infection with Babesia divergens-infected erythrocytes Saudi journal of Biological sciences (2010) 17, 129-132.

[21] Otsuka, Y.,Amasaki, M.,Yamato, O.,Maede,Y. Theeffectof macrophages on the erythrocytes oxidative damage and pathogenesis of anemia in babesia gibsoni infected dogs with low parasitemia J. Vet. Med. 2002 D. Sci. 64: 221-223.

[22] Mathe A,Voros K,Nemeth T,Biksi IHetyey C,Manczur F,Tekes L clinicopathological changes and effect of imidocarb therapy in dogs experimentally infected with Babesia canis Acta Vet Hung 2006 Mar; 54 (1): 19-33.

[23] Rees p, schoman JP plasma insulin concentrations in hypoglycemic dogs with Bbesia canis rossi infection Vet Parasitol 2008; 152: 60-66.

[24] Angel Tomas CAMACHOa, Francisco Javier GUITIANb, Estrella PALLASc, Juan Jesus GESTALd, Sonia OLMEDAe, Heidi GOETHERTf, g, Sam TELFORD IIIf,g, Andrew SPIELMANf. Serum protein response and renal failure in canine Babesia annae infection Vet. Res. 36 (2005) 713-722 713.

[25] Okubo K, Yokoyama N, Takabatake N, Okamura M, Igarashi I.2007, Amount of cholesterol in host membrane affects erythrocyte invasion and replication by Babesia bovis, Parasitology. 2007 May; 134 (Pt 5): 625-30. Epub 2006 Dec 6. 
[26] Bijan Esmaeilnejad, Mousa Tavassoli, Siamak Asri-Rezaei, Invistigation of hematological and biochemical parameters in small ruminants naturally infected with Babesia ovis Vetrinary Research Forum. 2012; 3 (1) 31-36.

[27] El Moghazy F.M, Impact of parasitic infection on Ovaraian Activity in Buffaloes-Heifers with Emphasis on Ascariasis, World journal of Zoology 6 (2): 196-203,2011.

[28] Sumamsetty, MD, PhD. zena khalil MD, Pamela schori, MT (ASCP), migul Azar, MD. Babisiosis two Atypical cases from Minnestota and review, Am. J. clin. pathol. 2003: 120: 554-559.

[29] White DJ, Talarico J,chang HG, Birkhead GS, Heimberger Tm Morse DL. Human babesiosis in New York state: Review of 139 hospitalized cases and analysis of prognostic factors. Arch intern Med. 1998; 158: 2149-54.

[30] Camacho At,Guitian FJ,Pallas E, et. al. Serum protein response and renal failure in canine babesia annae infection Vet. Res. 2005, 36, 713-722.

[31] Mathe A, Dobos, Kovacs M,Voros K, Histological and ultrastructure studies of renal lesions in Babesia canis infected dogs treated with imidocarb, Acta Vet Hung 2007 Dec; 55 (4): 511-23.

[32] Wittner M,Rowin KS, TanowitzHB, Hobbs jf, SsltzmanS, WenzB. Successful chemotherapy of transfusion babesiosis. Ann Intern Med. 1982, 96, 601-4.

[33] Rowin KS, Tanoitz HB, Wittner M. therapy of experimental babesiosis. Ann Intern Med. 1982, 97, 556-8.

[34] Krause PJ, Spielman Am Telford SR $3^{\text {rd }}$, Sikand VK, McKay K, Chrisianson D, et al. Persistent parasitemia after acute babesiosis. N Engl J Med. 1998; 339: 160-5.

[35] Pezhorn Bl, Lewis BD, Lopez-Rebolla LM and Swan GE, Tydskr S.S, nscreening of five drugs for efficacy against babesia felis in experimentally infected, South Africa Vet. Ass. 2000, 71: 1: 53-57.

[36] Marcia L.Buck., pharm.D., FccP. Use of clindamycin in pediatric infection volume 14 number 2 february 2008.

[37] Dorota M. Radomska-Leśniewska, Ewa Skopińska-Różewska, Jacek Malejczyk, Lincocin Experimental immunology. The effect of clindamycin and lincomycin on angiogenic activity of human blood mononuclear cells Centre Eur J Immunol 2010, 35 (4) 217222. 